\title{
GMR
}

\section{Isolation and characterization of twenty-three microsatellite loci for the black rockfish, Sebastes schlegelii}

\author{
F. Wang ${ }^{1}$, H. B. Jiang ${ }^{1}$, C. H. Han $^{3,4}$, G. P Cui ${ }^{1}$, C. F. Jia ${ }^{2 *}$, H.T. Ma ${ }^{3 * 4}$ \\ ${ }^{1}$ Shandong Provincial Key Laboratory of Restoration for Marine Ecology, Shandong \\ Marine Resource and Environment Research Institute, Yantai, P.R. China \\ ${ }^{2}$ Jiangsu Marine Fisheries Research Institute, Nantong, P.R. China \\ ${ }^{3}$ Key Laboratory of Tropical Marine Bio-resources and Ecology, Guangdong \\ Provincial Key Laboratory of Applied Marine Biology, South China Sea Institute of \\ Oceanology, Chinese Academy of Sciences, Guangzhou, P.R. China \\ ${ }^{4}$ South China Sea Bio-Resource Exploitation and Utilization Collaborative Innovation \\ Center, Guangzhou, P.R. China \\ Corresponding author: Chaofeng Jia \\ E-mail: chaofeng.124@163.com
}

Genet. Mol. Res. 17 (2): gmr16039912

Received Apr 02, 2018

Accepted May 01, 2018

Published May 05, 2018

DOI: http://dx.doi.org/10.4238/gmr16039912

Copyright (C) 2018 The Authors. This is an open-access article distributed under the terms of the Creative Commons Attribution ShareAlike (CC BY-SA) 4.0 License.

\begin{abstract}
Twenty-three polymorphic microsatellite loci were developed from the black rockfish, Sebastes schlegelii, with an enriched partial genomic library by magnetic beads and polymorphism of these loci was assessed in 32 individuals from a wild population. The loci yielded 2-19 alleles per locus, the observed, expected heterozygosity and polymorphic information content ranged from 0.063 to $1.000,0.091$ to 0.945 and 0.085 to 0.926, respectively. Twenty loci confirmed to Hardy-Weinberg equilibrium and only one pairs of loci show significant linkage disequilibrium after Bonferroni's correction. The availability of these markers will facilitate studies of the conservation genetics of $\mathrm{S}$. schlegelii.
\end{abstract}

Key words: Sebastes schlegelii; Microsatellite loci; Polymorphism 


\section{INTRODUCTION}

The black rockfish (S. schlegelii) is an important commercial fish distributed in Northwest Pacific enjoying high popularity in China, Korea and Japan (Yoshida et al., 2005). In recent decades, commercial exploitation and environmental changes have caused a decline in its population. Thus, demand for hypervariable molecular markers to provide a population-genetic perspective on conservation and management efforts of the spices (FAO, 1993) becomes urgent. Although few microsatellite loci have been isolated from S. schlegelii, more microsatellite markers are needed for further work on population structure within and among populations. In this paper, we describe twenty-three more polymorphic microsatellite loci that would be useful to facilitate further conservation genetics studies in S. schlegelii.

\section{MATERIAL AND METHODS}

Thirty-two individuals of $S$. schlegeli were collected from the northern coastal waters of the Yellow Sea and preserved in alcohol until DNA extraction. Genomic DNA was extracted from muscle using a regular phenolchloroform procedure (Sambrook et al., 2000), and simultaneously digested with restriction enzyme Sau3AI (Promega). DNA fragments ranging in size from 400 to $1000 \mathrm{bp}$ were collected and consequently ligated to adaptors (OligoA: 5'-GATCGTCGACGGTACCGAATTCT-3' and OligoB: 5'GTCAAGAATTCGGTACCGTCGAC-3'). DNA fragments were amplified in a $25-\mu \mathrm{L}$ reaction mix using the adaptor-specific primer (OligoA). Subsequently, the amplified DNA fragments were hybridized to biotinylated $(\mathrm{CA})_{16}$ and $(\mathrm{GA})_{16}$ probes, then captured by streptavidin-coated magnetic beads (Promega). Unhybridized DNA was washed away, and the remaining DNA was eluted from the beads. The enriched DNA was then ligated to pGEM-T easy vectors (Promega) and transformed into competent Escherichia coli DH5 $\alpha$ cells to construct an enriched microsatellite sequence library. Positive clones were identified by PCR with SP6, T7 and $(\mathrm{CA})_{16}$ or $(\mathrm{GA})_{16}$ primers and sequenced on an ABI 3730 XL DNA sequencer (Applied Biosystems).

Primers were designed using Primer Primer 5.0 (Premier Biosoft) and subsequently tested at standard PCR conditions with six specimens. Successfully amplified loci with expected sizes were further evaluated with a sample of thirty-two individuals collected from Changdao, Shandong Province. PCR reactions were performed in a $25-\mu \mathrm{L}$ reaction volume containing $0.4 \mu \mathrm{M}$ of each primer, $0.2 \mathrm{mM} \mathrm{dNTPs}, 2 \mathrm{mM} \mathrm{MgCl} 2,1 \times \mathrm{PCR}$ buffer, $1 \mathrm{U}$ Taq polymerase (Tiangen, China) and 50-100ng DNA. Amplification was performed under the following conditions: $94^{\circ} \mathrm{C}$ for $5 \mathrm{~min}$, followed by 32 cycles of $94^{\circ} \mathrm{C}$ for $45 \mathrm{~s}$, optimal annealing temperature (Table 1) for $45 \mathrm{~s}$, and $72^{\circ} \mathrm{C}$ for $1 \mathrm{~min}$, and a final extension step at $72^{\circ} \mathrm{C}$ for $10 \mathrm{~min}$. Amplified products were separated by electrophoresis on $8 \%$ non-denaturing polyacrylamide gels, and visualized with silver staining. Allele sizes were estimated according to a 50-bp DNA ladder (Tiangen, China) with software Gel-Pro Analyzer4.5 (Media Cybernetics).

The number of alleles $\left(N_{\mathrm{a}}\right)$, observed and expected heterozygosity $\left(H_{\mathrm{o}}, H_{\mathrm{e}}\right)$ were calculated using POPGENE 1.31 software (Yeh et al., 1999). Polymorphism information content (PIC) was estimated with PIC-CALC v0.6. Tests of Hardy-Weinberg equilibrium (HWE) and linkage disequilibrium (LD) were analyzed using GENEPOP v3.4 (Raymond and Rousset, 1995). The value for all diversity tests of significance was corrected by Bonferroni's correction (Rice, 1989).

\section{RESULTS AND DISCUSSION}

Sixty-eight positive clones were obtained after amplifying. Fifty-six of them contained microsatellites and Fiftytwo sequences provided sufficient quality for primer pairs to be designed after sequencing. Of the primers designed, 48 generated clear and specific products consistently, and 23 primers present polymorphic. A total of 151 alleles were detected in all 32 individuals. The allele number of the 23 polymorphic loci ranges from 2 to 19 with an average of 6.565 per locus. Polymorphism information content ranges from 0.085 to 0.926 .8 and 12 loci presented a medium or high level of polymorphism according to standard of Botstein et al. (1980), respectively. The observed and expected heterozygosities ranged from 0.063 to 1.000 (mean 0.503) and 0.091 to 0.945 (mean 0.599), respectively (Table 1). 
Table 1. Characteristics of twenty-three microsatellite loci developed from S. schlegelii.

\begin{tabular}{|c|c|c|c|c|c|c|c|c|c|}
\hline $\begin{array}{l}\text { Locus/ } \\
\text { Accessio } \\
\text { n No. }\end{array}$ & Primer sequence $\left(5^{\prime}-3^{\prime}\right)$ & $\begin{array}{l}\text { Ta } \\
\left({ }^{\circ} \mathrm{C}\right. \\
)\end{array}$ & Repeat motif & $\begin{array}{c}\text { Size } \\
\text { rang } \\
\mathbf{e} \\
\text { (bp) }\end{array}$ & $A$ & Ho & He & $P I C$ & $\begin{array}{c}\text { HW } \\
\mathrm{E} \\
\boldsymbol{P} \\
\text { value }\end{array}$ \\
\hline $\begin{array}{c}\text { HJ3-3/ } \\
\text { KJ472145 }\end{array}$ & $\begin{array}{l}\text { F:TGAGACTGAATTATGGGAGA } \\
\text { R:AAATGCTTACAGGGCTTC }\end{array}$ & 60 & $(\mathrm{AG})_{7}(\mathrm{TC})_{10}(\mathrm{AC})_{5}$ & $\begin{array}{l}122- \\
134\end{array}$ & 8 & $\begin{array}{c}0.18 \\
8\end{array}$ & $\begin{array}{c}0.86 \\
7\end{array}$ & $\begin{array}{c}0.83 \\
6\end{array}$ & $0.000^{*}$ \\
\hline $\begin{array}{c}\text { HJ5-1/ } \\
\text { KJ472146 }\end{array}$ & $\begin{array}{l}\text { F:ATACGCTCTGTATTCAACG } \\
\text { R:ACTTCCACATCAAATGTCCT }\end{array}$ & 63 & $(\mathrm{AG})_{7} \mathrm{C}(\mathrm{GA})_{19}$ & $\begin{array}{l}215- \\
267\end{array}$ & $\begin{array}{l}1 \\
2\end{array}$ & $\begin{array}{c}0.68 \\
8\end{array}$ & $\begin{array}{c}0.85 \\
9\end{array}$ & $\begin{array}{c}0.82 \\
8\end{array}$ & 0.062 \\
\hline $\begin{array}{c}\text { HJ5-6/ } \\
\text { KJ472147 }\end{array}$ & $\begin{array}{l}\text { F:CTGCCGAATCAGAGGTGT } \\
\text { R:GCCAGCGTAGGAAGGAAT }\end{array}$ & 60 & $(\mathrm{CT})_{10}$ & $\begin{array}{c}302- \\
311\end{array}$ & 4 & $\begin{array}{c}0.15 \\
6\end{array}$ & $\begin{array}{c}0.20 \\
5\end{array}$ & $\begin{array}{c}0.19 \\
3\end{array}$ & 0.030 \\
\hline $\begin{array}{c}\text { HJ5-7/ } \\
\text { KJ472148 }\end{array}$ & $\begin{array}{l}\text { F:CCCTTAGGCTGGACAACT } \\
\text { R:GTGAATCTGCACCCGTGA }\end{array}$ & 64 & $(\mathrm{CA})_{12} \mathrm{GA}(\mathrm{CA})_{7} \ldots(\mathrm{AC})_{5} \ldots(\mathrm{TG})_{5}$ & $\begin{array}{c}352- \\
363\end{array}$ & 6 & $\begin{array}{c}0.37 \\
5\end{array}$ & $\begin{array}{c}0.44 \\
5\end{array}$ & $\begin{array}{c}0.41 \\
4\end{array}$ & 0.029 \\
\hline $\begin{array}{c}\text { HJ5-24/ } \\
\text { KJ472149 }\end{array}$ & $\begin{array}{l}\text { F:AAATGAACTACCAAAGGAAGC } \\
\text { G } \\
\text { R:GGGAAATCTCAAAGCAAACAC } \\
\text { T }\end{array}$ & 64 & $(\mathrm{TTC})_{4} \ldots(\mathrm{GCT})_{5}$ & $\begin{array}{c}457- \\
468\end{array}$ & 4 & $\begin{array}{c}0.40 \\
6\end{array}$ & $\begin{array}{c}0.62 \\
2\end{array}$ & $\begin{array}{c}0.54 \\
7\end{array}$ & $0.000^{*}$ \\
\hline $\begin{array}{l}\text { HJ5-26/ } \\
\text { KJ472150 }\end{array}$ & $\begin{array}{l}\text { F:GACTCACCCTGCAAACAC } \\
\text { R:TACCACCCAGACACCACA }\end{array}$ & 64 & $(\mathrm{AC})_{13}$ & $\begin{array}{l}206- \\
232\end{array}$ & 9 & $\begin{array}{c}1.00 \\
0\end{array}$ & $\begin{array}{c}0.86 \\
9\end{array}$ & $\begin{array}{c}0.83 \\
9\end{array}$ & 0.010 \\
\hline $\begin{array}{l}\text { HJ5-29/ } \\
\text { KJ472151 }\end{array}$ & $\begin{array}{l}\text { F:ATGGCGTCTTGCCCTTGT } \\
\text { R:CGCGGCCTTTTCATCTCA }\end{array}$ & 60 & $(\mathrm{AC})_{8}$ & $\begin{array}{l}187- \\
201\end{array}$ & 9 & $\begin{array}{c}1.00 \\
0\end{array}$ & $\begin{array}{c}0.88 \\
1\end{array}$ & $\begin{array}{c}0.85 \\
3\end{array}$ & 0.007 \\
\hline $\begin{array}{c}\text { HJ5-31/ } \\
\text { KJ472152 }\end{array}$ & $\begin{array}{l}\text { F:AATTGGTTGTTAATTCCGGTCC } \\
\text { R:GCCTTTCTTTCCAAATTCAGTG }\end{array}$ & 50 & $(\mathrm{CT})_{26} \ldots(\mathrm{TC})_{5}$ & $\begin{array}{c}138- \\
145\end{array}$ & 2 & $\begin{array}{c}0.09 \\
4\end{array}$ & $\begin{array}{c}0.09 \\
1\end{array}$ & $\begin{array}{c}0.08 \\
5\end{array}$ & 1.000 \\
\hline $\begin{array}{c}\text { HJ5-34/ } \\
\text { KJ472153 }\end{array}$ & $\begin{array}{l}\text { F:TTCTGCTGAATGAGGGAG } \\
\text { R:CAGCGGGAGTTTGTATTG }\end{array}$ & 60 & $(\mathrm{AG})_{5}$ & $\begin{array}{c}490- \\
498\end{array}$ & 4 & $\begin{array}{c}0.50 \\
0\end{array}$ & $\begin{array}{c}0.53 \\
8\end{array}$ & $\begin{array}{c}0.49 \\
4\end{array}$ & 0.014 \\
\hline $\begin{array}{c}\text { HJ5-44/ } \\
\text { KJ472154 }\end{array}$ & $\begin{array}{l}\text { F:GCACCAATCTGTACCATCA } \\
\text { R:TAAGAGCCCAGTCACCAT }\end{array}$ & 60 & $(\mathrm{TGCG})_{3} \ldots(\mathrm{TGCG})_{3}$ & $\begin{array}{c}144- \\
166\end{array}$ & 6 & $\begin{array}{c}0.75 \\
0\end{array}$ & $\begin{array}{c}0.76 \\
6\end{array}$ & $\begin{array}{c}0.71 \\
6\end{array}$ & 0.179 \\
\hline $\begin{array}{l}\text { HJ5-46/ } \\
\text { KJ472155 }\end{array}$ & $\begin{array}{l}\text { F:CCACTGGCAGATAAACGA } \\
\text { R:TTTTAACGGGCAGTTGTG }\end{array}$ & 63 & $(\mathrm{TG})_{15} \ldots(\mathrm{TG})_{10}$ & $\begin{array}{l}197- \\
219\end{array}$ & $\begin{array}{l}1 \\
3\end{array}$ & $\begin{array}{c}0.65 \\
6\end{array}$ & $\begin{array}{c}0.85 \\
6\end{array}$ & $\begin{array}{c}0.82 \\
9\end{array}$ & 0.049 \\
\hline $\begin{array}{c}\text { HJ5-47/ } \\
\text { KJ472156 }\end{array}$ & $\begin{array}{l}\text { F:GGCCTCAAATGACAAGGT } \\
\text { R:CCAGGGTGCTCTGAAGAA }\end{array}$ & 63 & $(\mathrm{AC})_{5} \mathrm{G}(\mathrm{CA})_{20}$ & $\begin{array}{c}362- \\
496\end{array}$ & $\begin{array}{l}1 \\
9\end{array}$ & $\begin{array}{c}1.00 \\
0\end{array}$ & $\begin{array}{c}0.94 \\
5\end{array}$ & $\begin{array}{c}0.92 \\
6\end{array}$ & 0.114 \\
\hline $\begin{array}{c}\text { HJ5-58/ } \\
\text { KJ472157 }\end{array}$ & $\begin{array}{l}\text { F:CGCTCGGTGTTGATTTCG } \\
\text { R:CGCCACTTCAGCTCCTTT }\end{array}$ & 60 & $(\mathrm{CA})_{5}$ & $\begin{array}{c}210- \\
239\end{array}$ & 5 & $\begin{array}{c}0.37 \\
5\end{array}$ & $\begin{array}{c}0.39 \\
7\end{array}$ & $\begin{array}{c}0.36 \\
4\end{array}$ & 0.241 \\
\hline $\begin{array}{c}\text { HJ5-64/ } \\
\text { KJ472158 }\end{array}$ & $\begin{array}{l}\text { F:GTCCACTTGGTGAGGGGTTT } \\
\text { R:TGGAGCAGAACAGGGCGTAG }\end{array}$ & 60 & $(\mathrm{CT})_{6} \ldots(\mathrm{CT})_{5}$ & $\begin{array}{c}496- \\
500\end{array}$ & 3 & $\begin{array}{c}0.43 \\
8\end{array}$ & $\begin{array}{c}0.67 \\
3\end{array}$ & $\begin{array}{c}0.58 \\
8\end{array}$ & 0.007 \\
\hline $\begin{array}{c}\text { HJ5-65/ } \\
\text { KJ472159 }\end{array}$ & $\begin{array}{l}\text { F:TTTACAGGTCTTGAAGCAGG } \\
\text { R:GAAGTTGAAGTTTTGAGGG }\end{array}$ & 60 & $(\mathrm{CT})_{10}$ & $\begin{array}{c}176- \\
188\end{array}$ & 4 & $\begin{array}{c}0.50 \\
0\end{array}$ & $\begin{array}{c}0.66 \\
3\end{array}$ & $\begin{array}{c}0.58 \\
6\end{array}$ & 0.093 \\
\hline $\begin{array}{c}\text { HJ5-75/ } \\
\text { KJ472160 }\end{array}$ & $\begin{array}{l}\text { F:CACCTGGATTTATTTGGG } \\
\text { R:AAGAGGCAGTATGGATGG }\end{array}$ & 60 & $(\mathrm{CT})_{9}$ & $\begin{array}{c}168- \\
180\end{array}$ & 6 & $\begin{array}{c}0.37 \\
5\end{array}$ & $\begin{array}{c}0.47 \\
3\end{array}$ & $\begin{array}{c}0.44 \\
6\end{array}$ & 0.010 \\
\hline $\begin{array}{c}\text { HJ5-84/ } \\
\text { KJ472161 }\end{array}$ & $\begin{array}{l}\text { F:AGCATCAGGGATAAAGGA } \\
\text { R:GACGATTAGCCAGGTTGT }\end{array}$ & 60 & $(\mathrm{CA})_{6}$ & $\begin{array}{c}188- \\
199\end{array}$ & 5 & $\begin{array}{c}0.31 \\
3\end{array}$ & $\begin{array}{c}0.37 \\
9\end{array}$ & $\begin{array}{c}0.35 \\
3\end{array}$ & 0.027 \\
\hline $\begin{array}{l}\text { HJ5-103/ } \\
\text { KJ472162 }\end{array}$ & $\begin{array}{l}\text { F:ACAGGAGAACAGGCAAGG } \\
\text { R:TCAGCACGAGTACCGAAG }\end{array}$ & 62 & $(\mathrm{AC})_{27} \ldots(\mathrm{AC})_{12}$ & $\begin{array}{c}335- \\
411\end{array}$ & $\begin{array}{l}1 \\
0\end{array}$ & $\begin{array}{c}0.90 \\
6\end{array}$ & $\begin{array}{c}0.89 \\
5\end{array}$ & $\begin{array}{c}0.86 \\
9\end{array}$ & 0.118 \\
\hline $\begin{array}{l}\text { HJ5-120/ } \\
\text { KJ472163 }\end{array}$ & $\begin{array}{l}\text { F:GCCCGACTCACCAAAACA } \\
\text { R:TCCACAAATCGCCGTCTC }\end{array}$ & 52 & $(\mathrm{GA})_{6}$ & $\begin{array}{l}280- \\
285\end{array}$ & 3 & $\begin{array}{c}0.06 \\
3\end{array}$ & $\begin{array}{c}0.12 \\
1\end{array}$ & $\begin{array}{c}0.11 \\
6\end{array}$ & 0.031 \\
\hline $\begin{array}{l}\text { HJ5-134/ } \\
\text { KJ472164 }\end{array}$ & $\begin{array}{l}\text { F:AGTGAAGCCACTGTGAGA } \\
\text { R:AGTGAAGCCACTGTGAGA }\end{array}$ & 63 & $\begin{array}{l}(\text { TTAGGG })_{6} \text { GTT }(\text { GGGTTA })_{6} \ldots(\text { GGGTTA } \\
)_{5}\end{array}$ & $\begin{array}{c}126- \\
182\end{array}$ & 7 & $\begin{array}{c}0.71 \\
9\end{array}$ & $\begin{array}{c}0.82 \\
4\end{array}$ & $\begin{array}{c}0.78 \\
8\end{array}$ & 0.010 \\
\hline $\begin{array}{l}\text { HJ5-140/ } \\
\text { KJ472165 }\end{array}$ & $\begin{array}{l}\text { F:AAGAGCCCAGTCACCATG } \\
\text { R:TGACCTCGGGTAGAAACG }\end{array}$ & 60 & $(\mathrm{GCAC})_{4}$ & $\begin{array}{c}162- \\
186\end{array}$ & 4 & $\begin{array}{c}0.43 \\
8\end{array}$ & $\begin{array}{c}0.53 \\
4\end{array}$ & $\begin{array}{c}0.41 \\
7\end{array}$ & 0.323 \\
\hline $\begin{array}{l}\text { HJ5-153/ } \\
\text { KJ472166 }\end{array}$ & $\begin{array}{l}\text { F:TGCTGGATCGGTAAGAAG } \\
\text { R:CCCGCATCAGTGAGAAAA }\end{array}$ & 60 & $(\mathrm{TCCTC})_{3} \ldots(\mathrm{CT})_{7}$ & $\begin{array}{l}190- \\
200\end{array}$ & 4 & $\begin{array}{c}0.50 \\
0\end{array}$ & $\begin{array}{c}0.41 \\
1\end{array}$ & $\begin{array}{c}0.36 \\
8\end{array}$ & 0.774 \\
\hline $\begin{array}{l}\text { HJ5-154/ } \\
\text { KJ472167 }\end{array}$ & $\begin{array}{l}\text { F:GGGTGAGTGAGGAAGGAT } \\
\text { R:GCACAACTCTGCCAAAAC }\end{array}$ & 60 & $(\mathrm{CCTCT})_{4}$ & $\begin{array}{c}158- \\
178\end{array}$ & 4 & $\begin{array}{c}0.12 \\
5\end{array}$ & $\begin{array}{c}0.45 \\
5\end{array}$ & $\begin{array}{c}0.37 \\
2\end{array}$ & $0.000^{*}$ \\
\hline
\end{tabular}

\section{CONCLUSION}

After Bonferroni's correction, $p$ values were set to 0.002 for HWE and 0.0002 for linkage disequilibrium. Three loci (HJ3-3, HJ5-24 and HJ5-154) showed significant deviations in the observed genotype frequencies from Hardy-Weinberg equilibrium and one pair of loci yielded a significant linkage disequilibrium. The microsatellite loci developed here should be particularly useful for genetic identification and population management of $S$. schlegelii in future conservation biology efforts.

\section{ACKNOWLEDGMENTS}

This study was supported by China Agriculture Research System (CARS-47-Z14), Major Agriculture Application Technology Innovation Project of Shandong Province (2017-2020), Modern Agriculture Industry System of Shandong Province of China(SDAIT-22-04) and Science and Technology Planning Project of Guangdong Province,China (2017B030314052) 


\section{REFERENCES}

Botstein D, White RL, Skolnick M and Davis RW (1980). Construction of a genetic linkage map in man using restriction fragment length polymorphisms. Am J Hum Genet. 32: 314-331. https://doi.org/10.1007/bf00266542

FAO (1993). Report of the expert consultation on utilization and conservation of aquatic genetic resources. FAO Fish Rep. 491: 1-58.

Raymond M and Rousset F (1995). GENEPOP (version 1.2): Population genetics software for exact tests and ecumenicism. J Hered. 86 (3): 248-249. https://doi.org/10.1093/oxfordjournals.jhered.a111573

Rice WR (1989). Analyzing tables of statistical tests. Evolution. 43(1): 223-225. https://doi.org/10.2307/2409177

Sambrook JD and Russell W (2000). Molecular cloning: A laboratory manual, 2nd edn. Cold Spring Harbor Laboratory Press, Cold Spring

Harbor, New York. https://doi.org/10.1002/abio.370050118

Yeh FC, Yang RC and Boyle T (1999). Microsoft Window-based freeware for population genetic analysis (POPGENE), Ver. 1.31. University of Alberta, Canada.

Yoshida K, Nakagawa M and Wada S (2005). Multiplex PCR system applied for analyzing microsatellite loci of Schlegel's black rockfish, Sebastes schlegeli. Mol Ecol Res. 5 (2): 416-418. https://doi.org/10.1111/j.1471-8286.2005.00945.x 